\title{
Color vision impairment in multiple sclerosis and neuromyelitis optica spectrum disorder
}

\author{
Małgorzata Rogaczewska', Sławomir Michalak' ${ }^{2}$, Marcin Stopa' \\ 'Department of Ophthalmology, Chair of Ophthalmology and Optometry, Poznan University of Medical Sciences, Poznan, Poland \\ 2Department of Neurochemistry and Neuropathology, Chair of Neurology, Poznan University of Medical Sciences, Poznan, Poland
}

\begin{abstract}
Aim of the study: To evaluate the color vision impairment in multiple sclerosis (MS) and neuromyelitis optica spectrum disorder (NMOSD) and its relation to retinal neurovascular parameters measured by spectral-domain optical coherence tomography (SDOCT) and OCT angiography (OCTA).

Material and methods: A total of 40 relapsing-remitting MS patients, 13 aquaporin- 4 antibody-positive NMOSD patients, and 20 healthy controls were included. MS and NMOSD were divided into groups: eyes with a history of optic neuritis (MS+ON, $\mathrm{NMOSD}+\mathrm{ON}$ ) and eyes without previous ON (MS-ON, NMOSD-ON). Color vision was assessed with the FarnsworthMunsell 100 hue test and results were presented as the square root of total error score $(\sqrt{ } \mathrm{TES})$ and total partial error score $(\sqrt{ } \mathrm{TPES})$ for the blue-yellow (B-Y) and red-green (R-G) axes. Ganglion cell complex (GCC) and retinal nerve fiber layer (RNFL) thickness was obtained with SD-OCT and vessel density of superficial, deep, and radial peripapillary capillary plexuses (SCP, DCP, RPC) was obtained with OCTA.
\end{abstract}

Results: $\sqrt{T E S}, \mathrm{~B}-\mathrm{Y}$, and R-G $\sqrt{ }$ TPES were significantly higher in $\mathrm{MS}+\mathrm{ON}, \mathrm{MS}-\mathrm{ON}, \mathrm{NMOSD}+\mathrm{ON}$, and NMOSD-ON than in the control group. Color vision was comparable between $\mathrm{MS}+\mathrm{ON}$ and MS-ON, whereas in NMOSD+ON, it was significantly more impaired than in NMOSD-ON and MS+ON. Within the groups, the severity of dyschromatopsia did not differ between the B-Y and R-G axes. $\sqrt{T E S}$ correlated with GCC thickness only in NMOSD-ON eyes, while in MS+ON, an association between $\sqrt{T E S}$ and vessel density of SCP and RPC was observed. Color vision did not correlate with RNFL thickness and vessel density of DCP in any group.

Conclusions: Color vision defects were found in MS and NMOSD eyes, regardless of the history of ON. No specific predilection towards the blue-yellow or red-green axis was detected.

KEY WORDS: color vision, dyschromatopsia, multiple sclerosis, neuromyelitis optica spectrum disorder, optic neuritis, optical coherence tomography.

\section{INTRODUCTION}

Multiple sclerosis (MS) and neuromyelitis optica spectrum disorder (NMOSD) are inflammatory demyelinating conditions of the central nervous system $[1,2]$. Neuromyelitis optica spectrum disorder was considered a variant of MS until 2004, when the disease-specific serum IgG was discovered [3]. This antibody, which binds to the aquaporin-4 (AQP4) water channel presented in the membrane of astrocytes and Müller cells, can be detected in up to $80 \%$ of NMOSD patients $[4,5]$.

Multiple sclerosis and NMOSD patients had the color vision independently assessed with a variety of tests, inter alia, Ishihara plates, Hardy-Rand-Rittler (HRR) plates, Farnsworth Panel D-15, and the Farnsworth-Munsell 100 (FM-100) hue test [6-19]. Although time-consuming, the FM-100 hue test is considered the most useful in evaluating the acquired color defects by providing the severity and the type of dyschromatopsia [20].

Optic neuritis $(\mathrm{ON})$ is a common manifestation of both diseases, yet in NMOSD patients, the inflammation has a more severe course with a poorer visual outcome [21]. Regarding $\mathrm{ON}$ and non-ON eyes, the color vision abnormalities in MS and NMOSD patients were comparatively evaluated only in one study by Merle et al. [22]. They reported that the severity of dyschromatopsia did not differ between MS and NMOSD. However, the type of color defect was not defined [22].

Several authors have investigated the association between color vision impairment and retinal nerve fiber

\section{CORRESPONDING AUTHOR}

Marcin Stopa, MD, PhD, FEBO, Department of Ophthalmology, Chair of Ophthalmology and Optometry, Poznan University of Medical Sciences, 16/18 Grunwaldzka St., 60-780, Poznan, Poland, e-mail: stopa@ump.edu.pl 
layer (RNFL) and ganglion cell complex (GCC) thickness acquired with optical coherence tomography (OCT). In MS patients, the results were not consistent depending on the used test (HRR, Farnsworth Panel D-15, or FM-100), whereas in NMOSD patients, no correlation was found [7$13,18,19,23]$.

This study aimed to identify color vision abnormalities with the FM-100 hue test in MS and NMOSD patients, considering $\mathrm{ON}$ and non-ON eyes. Furthermore, we evaluated the relationship between the severity of dyschromatopsia and retinal neurovascular parameters obtained with spectral-domain OCT (SD-OCT) and OCT angiography (OCTA).

\section{MATERIAL AND METHODS}

\section{Ethical approval}

The study was performed in accordance with the Declaration of Helsinki and was approved by the medical ethics committee of Poznan University of Medical Sciences (approval no. 562/18 from May 2018). Written informed consent was obtained from all participants after a full explanation of the study.

\section{Study participants}

This single-center study was conducted at the Department of Ophthalmology and the Department of Neurology of Poznan University of Medical Sciences between June 2018 and September 2020. We recruited relapsing-remitting MS and AQP4-IgG seropositive NMOSD patients, as well as age- and sex-matched healthy controls. Multiple sclerosis and NMOSD were diagnosed according to the 2017 McDonald criteria and 2015 International Panel for NMO Diagnosis criteria, respectively $[24,25]$. The AQP4-IgG was detected by a commercial cell-based indirect immunofluorescence assay (EUROIMMUN AG, Lübeck, Germany). Analyses were performed in the Department of Neurochemistry and Neuropathology at Poznan University of Medical Sciences, which participates in an international external quality control system and receives regular certification for the detection of AQP4-IgG (Institut für Qualitätssicherung, Lübeck, Germany). Although, in total, 24 NMOSD patients were diagnosed with AQP4-IgG seropositive disease in our institution, only 13 patients were enrolled in this study. Seven patients could not undergo ophthalmic examination because of visual or physical disability, and four patients refused to participate. Clinical data, including the history of $\mathrm{ON}$ and disease duration, were collected. We classified MS and NMOSD patients' eyes based on the presence (MS+ON, $\mathrm{NMOSD}+\mathrm{ON}$ ) or absence (MS-ON, NMOSD-ON) of a previous $\mathrm{ON}$ episode.

The inclusion criteria were age $\geq 18$ years, no ON attack within the last 6 months before the examination, and at least 2 years of disease duration for MS patients. The exclusion criteria were myopia greater than 6 diopters, congenital dyschromatopsia, glaucoma, optic disc drusen, macular disease, hypertensive or diabetic retinopathy, history of uveitis, previous eye surgery, and low OCT image quality.

\section{Basic ophthalmic examination}

All participants underwent best-corrected visual acuity (BCVA) testing, slit-lamp biomicroscopy, indirect ophthalmoscopy, and Goldmann applanation tonometry with central corneal thickness correction. BCVA was assessed with the Early Treatment of Diabetic Retinopathy Study chart and expressed as a logarithm of the minimum angle of resolution $(\log M A R)$.

\section{Color vision assessment}

Color discrimination ability was evaluated by the FM-100 hue test (Farnsworth 100 Hue Test, Richmond Products Inc., Albuquerque, NM, USA) and carried out in a dark room under daylight illumination with a color temperature of 6280 kelvin (Daylight Illuminator, Good-Lite, Elgin, Il, USA). The test consists of 85 color caps that form a color circle and are stored in four boxes. Patients were asked to arrange the previously mixed-up caps in order according to hue between two fixed end caps in each box, with no time limit. Both eyes of the participants were tested monocularly, with the non-tested eye occluded.

For each cap, an error score was calculated as the sum of the differences between the number of that cap and the numbers of the two caps adjacent on each side. The total error score (TES) was computed as the sum of the error scores for each of the 85 caps. Additionally, we calculated the total partial error score (TPES) for the blue-yellow (B-Y) and redgreen (R-G) axes according to the method of Smith et al. [26]. As recommended, to reach the normal distribution of error scores for statistical analysis, the square root transformation

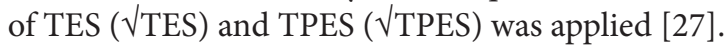

\section{OCT data acquisition}

The SD-OCT and OCTA images were obtained with RTVue XR Avanti with AngioVue (Optovue Inc., Fremont, CA, USA; software version 2017.1.0.151). The RNFL thickness was evaluated using the optic nerve head map protocol and measured at a diameter of $3.45 \mathrm{~mm}$ around the center of the optic disc. The GCC scan (7 x $7 \mathrm{~mm}$ square grid) was centered $1 \mathrm{~mm}$ temporal to the fovea, and the GCC thickness was automatically measured from the internal limiting membrane (ILM) to the outer boundary of the inner plexiform layer (IPL).

The AngioVue imaging system was based on a split-spectrum amplitude-decorrelation angiography algorithm that detects the blood motion in the vessels through sequentially obtained OCT cross-sectional scans. The blood flow maps presented the vessel density, i.e., the percentage area occupied by the perfused retinal blood vessels in the analyzed region $[28,29]$. The parafoveal retinal capillaries were visualized using a $3 \times 3 \mathrm{~mm}$ scan centered on the fovea and divided into plexuses by the AngioVue software. The superficial capillary plexus (SCP) was segmented from the ILM to $9 \mu \mathrm{m}$ above the IPL, and the deep capillary plexus (DCP) was segmented from $9 \mu \mathrm{m}$ above the IPL to $9 \mu \mathrm{m}$ below the outer plexiform layer. The parafovea was defined as an annulus with an inner 
and outer diameter of $1.0 \mathrm{~mm}$ and $3.0 \mathrm{~mm}$, respectively. The radial peripapillary capillary (RPC) plexus was acquired using $4.5 \times 4.5 \mathrm{~mm}$ rectangle scan centered on the optic nerve head. The capillaries within the ILM and the nerve fiber layer's outer boundary were analyzed in the peripapillary area defined as a $1.0-\mathrm{mm}$ wide round annulus extending outward from the optic disc boundary. Low-quality OCT images, i.e., with the signal strength index $<50$ or significant motion artifacts, were not analyzed. The OCT data were reported in alignment with APOSTEL recommendations [30].

\section{Statistical analysis}

Statistical analysis was performed using Statistica v13.3 (StatSoft, Inc., Tulsa, OK, USA) and SPSS (SPSS, Inc., Chicago, IL, USA). The Shapiro-Wilk test was used to determine the distribution of continuous variables. The differences between two unpaired groups were assessed using Student's $t$-test and the Mann-Whitney $U$-test, whereas the Chi-square test and Kruskal-Wallis test were used to evaluate the differences among several unpaired groups. We performed generalized estimating equation models accounting for intrasubject inter-eye dependencies to compare the color vision error score between cohorts. Pearson's or Spearman's correlations were calculated between $\sqrt{T E S}$ and selected variables. Due to the exploratory nature of this study, no correction for multiple comparisons was made. Statistical significance was set at $p<0.05$.

\section{RESULTS}

Study population

In total, $40 \mathrm{MS}$ patients, 13 NMOSD patients, and 20 healthy controls were enrolled. We evaluated eyes with the complete OCT data; therefore, seven eyes of MS patients and nine eyes of NMOSD patients were excluded. There was no difference between the groups regarding age, sex, and disease duration except for BCVA of enrolled eyes $(p=0.014)$. Demographic and clinical features are summarized in Table I.

\section{Color vision}

The $\sqrt{T E S}$, as well as B-Y and R-G $\sqrt{T P E S}$, were significantly higher in $\mathrm{MS}+\mathrm{ON}, \mathrm{MS}-\mathrm{ON}, \mathrm{NMOSD}+\mathrm{ON}$, and NMOSD-ON than in the control group (Tables II and III, Figure 1). Comparing $\mathrm{ON}$ and non-ON eyes, the color vision did not differ in MS eyes, whereas in NMOSD, the $\sqrt{T E S}$ and $\sqrt{T P E S}$ were significantly higher in ON eyes (Table III). The color vision was significantly more impaired in NMOS$\mathrm{D}+\mathrm{ON}$ than $\mathrm{MS}+\mathrm{ON}$ eyes, while between NMOSD-ON and MS-ON groups, the error scores were comparable (Table III). Within the groups, the severity of dyschromatopsia did not

Table I. Demographic and clinical characteristics of the study population

\begin{tabular}{|l|c|c|c|} 
& MS & NMOSD & Controls \\
\hline Number of subjects & 40 & 13 & 20 \\
\hline Number of eyes enrolled & 73 & 17 & 40 \\
\hline ON eyes & 30 & 8 & - \\
\hline Non-ON eyes & 43 & 9 & 40 \\
\hline Age (years), mean \pm SD & $35.15 \pm 7.47$ & $42.08 \pm 10.23$ & $37.90 \pm 11.47$ \\
\hline Sex (female/male) & $32 / 8$ & $11 / 2$ & $17 / 3$ \\
\hline Disease duration (years), median (range) & $8(3-32)$ & $9(1-33)$ & - \\
\hline BCVA of enrolled eyes (logMAR), median (range) & $0.00(0.00-0.20)$ & $0.00(0.00-0.20)$ & $0.00(0.00-0.00)$ \\
\hline
\end{tabular}

$B C V A$ - best-corrected visual acuity; logMAR - the logarithm of the minimum angle of resolution; MS - multiple sclerosis; NMOSD - neuromyelitis optica spectrum disorder; ON - optic neuritis; SD - standard deviation

Table II. Baseline color vision, spectral-domain OCT and OCT angiography results of patients and controls

\begin{tabular}{|l|c|c|c|c|c|c|c|c|}
\hline & \multicolumn{3}{|c|}{ FM-100 hue test } & \multicolumn{2}{c|}{ Spectral-domain OCT } & \multicolumn{3}{c|}{ OCT angiography } \\
\cline { 2 - 9 } & $\sqrt{ }$ TES & $\sqrt{ }$ TPES B-Y & $\sqrt{ }$ TPES R-G & GCC $(\mu \mathrm{m})$ & RNFL $(\mu \mathrm{m})$ & SCP $(\%)$ & DCP $(\%)$ \\
\hline MS+ON & $10.01 \pm 4.62$ & $7.14 \pm 3.47$ & $6.94 \pm 3.23$ & $83.27 \pm 8.74$ & $85.57 \pm 11.39$ & $43.48 \pm 4.50$ & $57.03 \pm 2.22$ & $46.76 \pm 4.96$ \\
\hline MS-ON & $8.56 \pm 3.35$ & $6.06 \pm 2.44$ & $5.98 \pm 2.43$ & $90.98 \pm 7.46$ & $92.33 \pm 10.23$ & $46.71 \pm 2.67$ & $55.76 \pm 2.17$ & $49.91 \pm 2.99$ \\
\hline NMOSD+ON & $13.76 \pm 4.31$ & $9.75 \pm 3.20$ & $9.65 \pm 3.10$ & $77.75 \pm 10.70$ & $76.63 \pm 14.60$ & $42.43 \pm 7.00$ & $55.91 \pm 2.29$ & $42.79 \pm 7.20$ \\
\hline NMOSD-ON & $9.35 \pm 3.96$ & $7.01 \pm 2.98$ & $6.15 \pm 2.69$ & $94.33 \pm 9.10$ & $100.11 \pm 10.71$ & $48.29 \pm 3.31$ & $55.46 \pm 1.50$ & $50.49 \pm 1.66$ \\
\hline Controls & $5.75 \pm 2.52$ & $4.23 \pm 1.99$ & $3.71 \pm 2.02$ & $99.15 \pm 5.11$ & $101.60 \pm 7.46$ & $49.96 \pm 2.50$ & $54.33 \pm 2.45$ & $52.97 \pm 2.51$ \\
\hline
\end{tabular}

Values are reported as mean \pm standard deviation. B-Y - blue-yellow axis; DCP - deep capillary plexus; FM-100 - Farnsworth-Munsell 100 hue test; GCC - ganglion cell complex; MS - multiple sclerosis; NMOSD - neuromyelitis optica spectrum disorder; OCT - optical coherence tomography; ON - optic neuritis; R-G - red-green axis; RNFL - retinal nerve fiber layer; RPC - radial peripapillary capillaries; SCP - superficial capillary plexus; TES - total error score; TPES - total partial error score 


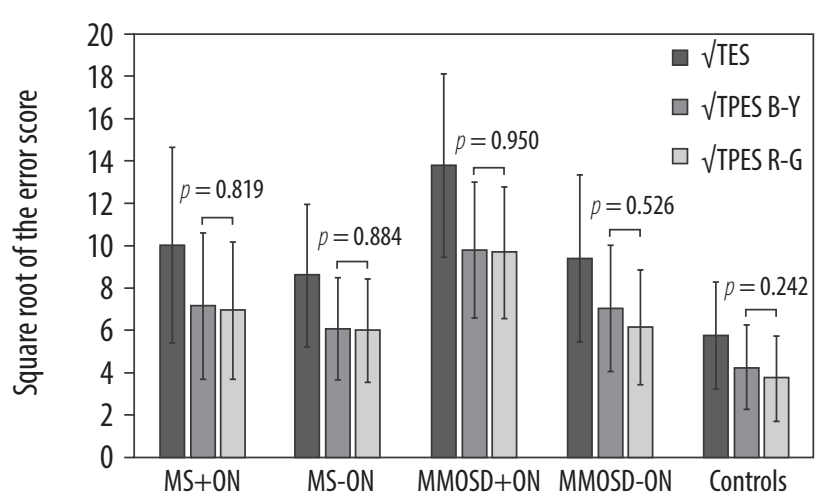

Figure 1. Bar plots illustrating the mean and standard deviation of the square root of total error score ( $\sqrt{ }$ TES) and total partial error score $(\sqrt{ }$ TPES) for blueyellow (B-Y) and red-green (R-G) axes. The B-Y $\sqrt{T P E S}$ and $R-G \sqrt{ }$ TPES were comparable within the groups

differ between the B-Y and R-G axes (Figure 1). The type of color vision defects was identified as follows: tritan in $\mathrm{MS}+\mathrm{ON}(n=2)$ and NMOSD+ON $(n=1)$ eyes, and pro$\tan$ in one MS-ON eye. The remaining eyes presented mixed color defects.

\section{Correlation between $\sqrt{ } \mathrm{TES}$ and clinical data}

A significant positive moderate correlation was found between $\sqrt{T E S}$ and age in MS+ON, MS-ON, and control groups (Table IV). No significant correlation between $\sqrt{T E S}$ and age was observed in NMOSD+ON $(r=-0.467, p=0.243)$ and NMOSD-ON ( $\mathrm{r}=-0.302, p=0.429$; Table IV). An association between $\sqrt{T E S}$ and disease duration was found in $\mathrm{MS}+\mathrm{ON}$ and MS-ON eyes only (Table IV).

\section{Correlation between $\sqrt{ }$ TES and OCT parameters}

The baseline SD-OCT and OCTA measurements are presented in Table II. The $\sqrt{T E S}$ strongly negatively correlated with GCC thickness in NMOSD-ON eyes $(r=-0.738$, $p=0.023$ ), whereas in other groups, this relationship was insignificant (Table IV). An association between $\sqrt{T E S}$ and the vessel density of SCP and RPC was only found in $\mathrm{MS}+\mathrm{ON}$ eyes $(\mathrm{r}=-0.518, p=0.003 ; \mathrm{r}=-0.413, p=0.023$, respectively; Table IV). The $\sqrt{T E S}$ did not correlate with RNFL thickness or the vessel density of DCP in any group (Table IV).

\section{DISCUSSION}

In this study, we found color vision impairment in MS and NMOSD patients in both ON and non-ON eyes. More-

Table III. Comparison of color vision error score between patients and controls and between selected groups

\begin{tabular}{|c|c|c|c|c|c|c|}
\hline & \multicolumn{3}{|c|}{ MS+0N vs. Controls } & \multicolumn{3}{|c|}{ MS+ON vs. MS-ON } \\
\hline & $\beta$ & $95 \% \mathrm{Cl}$ & $p$-value & $\beta$ & $95 \% \mathrm{Cl}$ & $p$-value \\
\hline$\sqrt{T E S}$ & 4.256 & (2.264 to 6.249 ) & $<0.001$ & 1.445 & $(-0.119$ to 3.009$)$ & 0.070 \\
\hline VTPES B-Y & 2.905 & (1.398 to 4.413) & $<0.001$ & 1.077 & $(-0.072$ to 2.226$)$ & 0.066 \\
\hline \multirow[t]{3}{*}{$\sqrt{ }$ TPES R-G } & 3.235 & $(1.779$ to 4.690$)$ & $<0.001$ & 0.956 & $(-0.194$ to 2.106$)$ & 0.103 \\
\hline & \multicolumn{3}{|c|}{ MS-ON vs. Controls } & \multicolumn{3}{|c|}{ NMOSD+ON vs NMOSD-ON } \\
\hline & $\beta$ & $95 \% \mathrm{Cl}$ & $p$-value & $\beta$ & $95 \% \mathrm{Cl}$ & $p$-value \\
\hline$\sqrt{T E S}$ & 2.811 & (1.287 to 4.335) & $<0.001$ & 4.410 & (1.882 to 6.937$)$ & $<0.001$ \\
\hline VTPES B-Y & 1.829 & (0.694 to 2.963) & 0.002 & 2.734 & (0.981 to 4.488$)$ & 0.002 \\
\hline \multirow[t]{3}{*}{$\sqrt{\text { TPES R-G }}$} & 2.279 & (1.109 to 3.448) & $<0.001$ & 3.503 & (1.534 to 5.472 ) & $<0.001$ \\
\hline & \multicolumn{3}{|c|}{ NMOSD+ON vs. Controls } & \multicolumn{3}{|c|}{ NMOSD+ON vs. MS+ON } \\
\hline & $\beta$ & $95 \% \mathrm{Cl}$ & $p$-value & $\beta$ & $95 \% \mathrm{Cl}$ & $p$-value \\
\hline$\sqrt{ } \mathrm{TES}$ & 8.004 & (5.081 to 10.927) & $<0.001$ & 3.748 & (0.530 to 6.966) & 0.022 \\
\hline VTPES B-Y & 5.516 & (3.294 to 7.737 ) & $<0.001$ & 2.610 & (0.180 to 5.041$)$ & 0.035 \\
\hline \multirow[t]{3}{*}{ VTPES R-G } & 5.944 & (3.872 to 8.015) & $<0.001$ & 2.709 & (0.466 to 4.952) & 0.018 \\
\hline & \multicolumn{3}{|c|}{ NMOSD-ON vs. Controls } & \multicolumn{3}{|c|}{ NMOSD-ON vs. MS-ON } \\
\hline & $\beta$ & $95 \% \mathrm{Cl}$ & $p$-value & $\beta$ & $95 \% \mathrm{Cl}$ & $p$-value \\
\hline$\sqrt{\mathrm{TES}}$ & 3.594 & (0.774 to 6.415$)$ & 0.012 & 0.783 & $(-2.066$ to 3.633$)$ & 0.590 \\
\hline VTPES B-Y & 2.781 & (0.659 to 4.904) & 0.010 & 0.953 & $(-1.167$ to 3.073$)$ & 0.378 \\
\hline VTPES R-G & 2.440 & (0.454 to 4.427) & 0.016 & 0.162 & $(-1.822$ to 2.146$)$ & 0.873 \\
\hline
\end{tabular}

$\beta$ - regression coefficient; $B$-Y - blue-yellow axis; C - confidence interval; MS - multiple sclerosis; NMOSD - neuromyelitis optica spectrum disorder; ON - optic neuritis; R-G - red-green axis; TES -total error score; TPES - total partial error score. 
Table IV. Correlation between color vision error score and age, disease duration, and OCT parameters

\begin{tabular}{|c|c|c|c|c|c|c|c|c|c|c|}
\hline & & & & & & & & & & \\
\hline & & & & & NMC & $-O N$ & NMC & ON & & \\
\hline & $r$ & $p$ & $r$ & $p$ & $r$ & $p$ & $r$ & $p$ & $r$ & $p$ \\
\hline Age & 0.488 & 0.006 & 0.489 & 0.001 & -0.467 & 0.243 & -0.302 & 0.429 & 0.455 & 0.003 \\
\hline Disease duration & 0.418 & 0.021 & 0.428 & 0.004 & 0.498 & 0.210 & 0.455 & 0.219 & - & - \\
\hline $\mathrm{GCC}$ & -0.323 & 0.082 & -0.236 & 0.128 & -0.437 & 0.279 & -0.738 & 0.023 & -0.114 & 0.483 \\
\hline RNFL & -0.241 & 0.200 & -0.211 & 0.174 & -0.297 & 0.475 & -0.665 & 0.051 & -0.172 & 0.289 \\
\hline$S C P$ & -0.518 & 0.003 & -0.203 & 0.192 & -0.652 & 0.080 & -0.472 & 0.199 & -0.191 & 0.238 \\
\hline$D C P$ & 0.168 & 0.376 & -0.146 & 0.349 & -0.184 & 0.663 & -0.007 & 0.987 & -0.212 & 0.189 \\
\hline $\mathrm{RPC}$ & -0.413 & 0.023 & -0.237 & 0.127 & -0.266 & 0.524 & 0.319 & 0.402 & 0.124 & 0.447 \\
\hline
\end{tabular}

DCP - deep capillary plexus; GCC - ganglion cell complex; MS - multiple sclerosis; NMOSD - neuromyelitis optica spectrum disorder; OCT - optical coherence tomography; ON - optic neuritis; RNFL - retinal nerve fiber layer; $R P C$ - radial peripapillary capillaries; SCP - superficial capillary plexus; TES - total error score

over, the in-depth analysis revealed no specific color vision pattern in those eyes.

There are only a few studies where the color vision was assessed with the FM-100 hue test in MS and NMOSD patients $[15-19,22,23]$. In two of them, the authors did not present the color vision abnormalities strictly associated with $\mathrm{ON}$, because the $\mathrm{ON}$ and non-ON eyes were reported as one group $[18,23]$. Two other studies evaluated only MS-ON eyes, and the results were inconsistent $[17,19]$. However, Fleishman et al. found that MS+ON and MS-ON groups had significantly higher TES than healthy controls, which was in agreement with our findings [15]. So far, little is known about the color vision defects in NMOSD. No study has examined the effect of optic neuritis on color discrimination ability in those patients. Our analysis showed that $\mathrm{ON}$ and non-ON eyes had higher error scores in NMOSD patients than the control group.

Comparing the severity of TPES, we found the errors evenly distributed between B-Y and R-G axes in MS and NMOSD groups. Additionally, the tritan and protan axis of confusion was observed only in three eyes (two MS+ON, one $\mathrm{NMOSD}+\mathrm{ON}$ ) and in one eye (one MS-ON), respectively. In 1995, Katz reported that no single type of dyschromatopsia occurred in MS+ON eyes [16]. Two recent studies employing Farnsworth Panel D-15 provided similar results, i.e., the mixed color deficiency was present in MS-ON and NMOSD-ON groups $[13,14]$. Consequently, our finding of no specific confusion axis was in line with the previous reports.

Our study showed that TES and TPES did not differ between MS+ON and MS-ON eyes, whereas the color vision in $\mathrm{NMOSD}+\mathrm{ON}$ was significantly more impaired than in NMOSD-ON and MS+ON eyes. We suppose that the comparable results between MS groups may be related to the progressive neurodegeneration process, which is $\mathrm{ON}$-independent and more pronounced in MS than in NMOSD [31]. In NMOSD, the inflammation of the optic nerve is more severe than in MS [21]. Thus, it may explain more significant color vision defects in NMOSD+ON compared with both
NMOSD-ON and MS+ON. As mentioned above, MS and NMOSD eyes have been collectively evaluated only in one study [22]. Merle et al. found no difference between either $\mathrm{MS}+\mathrm{ON}$ and NMOSD+ON or MS-ON and NMOSD-ON groups [22]. Likewise, in our study, the severity of color vision defects was similar between MS-ON and NMOSD-ON eyes.

It is known that in adults, the color discrimination ability deteriorates with age [32]. Such a relationship can be observed in our healthy controls. In both MS groups, we also noted a moderate positive correlation between TES and age, whereas in NMOSD+ON and NMOSD-ON the relationship was negative but not significant. According to disease duration, a moderate positive association with TES was observed in MS groups. These findings suggest that in NMOSD patients, severe color vision impairment may appear at a younger age or earlier in the disease course.

The link between color vision defects and retinal neuronal and axonal loss in MS has been a subject of several studies; however, only non-ON eyes were investigated $[8,9,13,19]$. Depending on the used test, the GCC and RNFL thickness correlated with color vision outcome (HRR, Farnsworth Panel D-15) or not (FM-100) $[8,9,13,19]$. Lampert et al. reported that HRR scores were strongly related to retinal ganglion cell injury in MS-ON eyes [9]. They also suggested that dyschromatopsia might serve as an accurate marker of retinal damage [9]. In fact, not every test is appropriate to be used in detailed analysis. The HRR test was designed for screening purposes, and, together with the Farnsworth Panel D-15, did not provide the precise assessment of color vision as did the FM-100 hue test [20]. In our study, the correlation between TES and GCC thickness was observed only in NMOSD-ON eyes, and RNFL thickness was not associated with TES in any group. Interestingly, we noted an association between color vision and vessel density of SCP and RPC in the MS+ON group. In $\mathrm{NMOSD}+\mathrm{ON}$, a similar moderate trend was found regarding SCP. We did not find any study showing such a relation; thus, we cannot clearly state whether this is an incidental or significant finding. 
Our study has several limitations. The group of NMOSD patients was relatively small due to the low prevalence of the disease. We evaluated only AQP4-IgG seropositive NMOSD patients. Therefore, the results may not extend to seronegative NMOSD. Because this was a single-center study, further investigations with different populations are necessary to confirm our observations.

In conclusion, our study revealed color vision impairment in MS and NMOSD patients, regardless of optic neuritis history. Moreover, no predilection towards the B-Y or R-G axis was found in those groups. Our results show that dyschro- matopsia was not associated with retinal ganglion cells and axonal damage.

\section{ACKNOWLEDGEMENTS}

We would like to thank Ass. Prof. Alicja Kalinowska-Łyszczarz and Dr. Michalina Jasiak-Zatońska for their assistance in patients' recruitment.

\section{DISCLOSURE}

The authors declare no conflict of interest.

\section{References}

1. Filippi M, Bar-Or A, Piehl F, et al. Multiple sclerosis. Nat Rev Dis Primers 2018; 4: 43.

2. Wingerchuk DM, Lennon VA, Lucchinetti CF, et al. The spectrum of neuromyelitis optica. Lancet Neurol 2007; 6: 805-815.

3. Lennon VA, Wingerchuk DM, Kryzer TJ, et al. A serum autoantibody marker of neuromyelitis optica: distinction from multiple sclerosis. Lancet 2004; 364: 2106-2112.

4. Jarius S, Wildemann B, Paul F. Neuromyelitis optica: clinical features, immunopathogenesis and treatment. Clin Exp Immunol 2014; 176: $149-164$.

5. Verkman AS, Ruiz-Ederra J, Levin MH. Functions of aquaporins in the eye. Prog Retin Eye Res 2008; 27: 420-433.

6. Sherif M, Bergin C, Borruat FX. Normal visual recovery after optic neuritis despite significant loss of retinal ganglion cells in patients with multiple sclerosis. Klin Monbl Augenheilkd 2019; 236: 425-428.

7. Villoslada P, Cuneo A, Gelfand J, et al. Color vision is strongly associated with retinal thinning in multiple sclerosis. Mult Scler 2012; 18: 991-999.

8. Martínez-Lapiscina EH, Ortiz-Pérez S, Fraga-Pumar E, et al. Colour vision impairment is associated with disease severity in multiple sclerosis. Mult Scler 2014; 20: 1207-1216.

9. Lampert EJ, Andorra M, Torres-Torres $\mathrm{R}$, et al. Color vision impairment in multiple sclerosis points to retinal ganglion cell damage. J Neurol 2015; 262: 2491-2497.

10. Felgueiras H, Parra J, Cruz S, et al. Dyschromatopsia in multiple sclerosis patients: a marker of subclinical involvement? J Neuroophthalmol 2016; 36: 275-279.

11. Sanchez-Dalmau B, Martinez-Lapiscina EH, Pulido-Valdeolivas I, et al. Predictors of vision impairment in multiple sclerosis. PLoS One 2018; 13: e0195856.

12. Satue M, Rodrigo MJ, Otin S, et al. Relationship between visual dysfunction and retinal changes in patients with multiple sclerosis. PLoS One 2016; 11: e0157293.

13. Barreiro-González A, Sanz MT, Carratalà-Boscà S, et al. Dyschromatopsia in multiple sclerosis reflects diffuse chronic neurodegeneration beyond anatomical landmarks. Acta Neurol Belg 2020; https://doi.org/10.1007/s13760-020-01516-x

14. Ong Chin Feng W, Wan Hitam WH. Evaluation of retinal nerve fiber layer thickness and optic nerve functions in fellow eye of neuromyelitis optica with unilateral optic neuritis. Taiwan J Ophthalmol 2020; 10: 189-196.

15. Fleishman JA, Beck RW, Linares OA, et al. Deficits in visual function after resolution of optic neuritis. Ophthalmology 1987; 94: 1029-1035.

16. Katz B. The dyschromatopsia of optic neuritis: a descriptive analysis of data from the optic neuritis treatment trial. Trans Am 0phthalmol Soc 1995; 93: 685-708.

17. Gundogan FC, Tas A, Altun S, et al. Color vision versus pattern visual evoked potentials in the assessment of subclinical optic pathway involvement in multiple sclerosis. Indian J Ophthalmol 2013; 61: 100-103.

18. Longbrake EE, Lancia S, Tutlam N, et al. Quantitative visual tests after poorly recovered optic neuritis due to multiple sclerosis. Mult Scler Relat Disord 2016; 10: 198-203.

19. Yuksel B, Dogan B, Koctekin B, et al. Color vision testing versus pattern visual evoked potentials and optical coherence tomography parameters in subclinical optic nerve involvement in multiple sclerosis. J Clin Neurosci 2019; 61: 48-53.

20. Melamud A, Hagstrom S, Traboulsi E. Color vision testing. Ophthalmic Genet 2004; 25: 159-187.

21. Srikajon J, Siritho S, Ngamsombat C, et al. Siriraj Neuroimmunology Research Group. Differences in clinical features between optic neuritis in neuromyelitis optica spectrum disorders and in multiple sclerosis. Mult Scler J Exp TransI Clin 2018; 4: 2055217318791196

22. Merle H, Olindo S, Jeannin S, et al. Visual field characteristics in neuromyelitis optica in absence of and after one episode of optic neuritis. Clin Ophthalmol 2013; 7: 1145-1153.

23. Merle $\mathrm{H}$, Olindo S, Donnio A, et al. Retinal peripapillary nerve fiber layer thickness in neuromyelitis optica. Invest Ophthalmol Vis Sci 2008; 49: 4412-4417.

24. Thompson AJ, Banwell BL, Barkhof F, et al. Diagnosis of multiple sclerosis: 2017 revisions of the McDonald criteria. Lancet Neurol 2018; 17: 162-173.

25. Wingerchuk DM, Banwell B, Bennett JL, et al. International Panel for NMO Diagnosis. International consensus diagnostic criteria for neuromyelitis optica spectrum disorders. Neurology 2015; 85: 177-189.

26. Smith VC, Pokorny J, Pass AS. Color-axis determination on the Farnsworth-Munsell 100-hue test. Am J Ophthalmol 1985; 100: 176182.

27. Aspinall PA. Inter-eye comparison on the 100 hue test. Acta Ophthalmol (Copenh) 1974; 52: 307-316.

28. Jia Y, Tan 0, Tokayer J, et al. Split-spectrum amplitude-decorrelation angiography with optical coherence tomography. Opt Express 2012; 20: 4710-4725. 
29. Ferrara D, Waheed NK, Duker JS. Investigating the choriocapillaris and choroidal vasculature with new optical coherence tomography technologies. Prog Retin Eye Res 2016; 52: 130-155.

30. Cruz-Herranz A, Balk LJ, Oberwahrenbrock T, et al. The APOSTEL recommendations for reporting quantitative optical coherence tomography studies. Neurology 2016; 86: 2303-9.

31. Kawachi I, Lassmann H. Neurodegeneration in multiple sclerosis and neuromyelitis optica. J Neurol Neurosurg Psychiatry 2017; 88: 137-145.

32. Verriest G, Van Laethem J, Uvijls A. A new assessment of the normal ranges of the Farnsworth-Munsell 100-hue test scores. Am J Ophthalmol 1982; 93: 635-642. 